\title{
One-Point Functions of $N=2$ Super-Liouville Theory with Boundary
}

Changrim Ahn, ${ }^{1}$ Marian Stanishkov, ${ }^{2}$ and Masayoshi Yamamoto ${ }^{3}$

\author{
Department of Physics \\ Ewha Womans University \\ Seoul 120-750, Korea \\ PACS: $11.25 . \mathrm{Hf}, 11.55 . \mathrm{Ds}$
}

\begin{abstract}
We derive one-point functions of the $N=2$ super-Liouville theory on a half line using the modular transformations of the characters in terms of the bulk and boundary cosmological constants. We also show that these results are consistent with conformal bootstrap equations which are based on the bulk and boundary actions. We provide various independent checks for our results.
\end{abstract}

\section{Introduction}

Two-dimensional Liouville field theory (LFT) has been studied for its relevance with non-critical string theories and two-dimensional quantum gravity [1, 2]. This theory has been extended to the supersymmetric Liouville field theories (SLFTs) which can describe the non-critical superstring theories. The LFT and SLFTs are irrational conformal field theories (CFTs) which have continuously infinite number of primary fields. It is very important to develope a CFT formalism which can apply to these irrational CFTs. There have been a lot of developments in this direction. Various methods have been proposed [3] to derive structure constants and reflection amplitudes, which are basic building blocks to complete the conformal bootstrap 4, 5. These have been extended to the $N=1$ SLFT in [6, 7].

More challenging problem is to extend these formalisms to the CFTs with a bound-

\footnotetext{
1ahn@ewha.ac.kr

${ }^{2}$ stanishkov@dante.ewha.ac.kr; On leave of absence from Institute of Nuclear Research and Nuclear Energy, Sofia, Bulgaria

3yamamoto@dante.ewha.ac.kr
} 
ary with a conformal boundary condition (BC). Cardy showed that the conformal BCs can be associated with the primary fields in terms of modular $S$-matrix elements for rational CFTs [8]. It has been an active issue to extend Cardy formalism to the irrational CFTs. For example, the conformally invariant boundary states can be related to D-branes in the context of string theories [9, 10, 11. An important progress in this direction has been made in [12]. With a boundary action which preserves the conformal symmetry, one-point function of a bulk operator and two-point correlation functions of boundary operators have been computed by conformal bootstrap method which extends the functional relation method developed for the bulk theory [4. A similar treatment of the LFT defined in the classical Lobachevskiy plane, namely the pseudosphere has been made in [13. This approach is generalized to the $N=1 \mathrm{SLFT}$ for the one-point functions [14] and the boundary two-point functions [15].

In this paper, we extend this approach to the $N=2$ SLFT. This theory is of a particular interest in the string community for rich properties [16]. In spite of the extended symmetry, it turns out that exact correlation functions of the $N=2$ SLFT are much more difficult to derive than previous cases. The main reason is that the $N=2$ SLFT has no strong-weak coupling duality. The invariance of the LFT and $N=1$ SLFT under $b \rightarrow 1 / b$ is realized when the background charge changes to $1 / b+b$ from its classical value of $1 / b$ after quantum corrections [2, 17. All the physical quantities like the correlations functions depend on the coupling constant through this combination. This invariance maintains an equivalence between a weak $b<<1$ and strong $b>>1$ coupling limits. This duality as well as the functional relations based on the conformal bootstrap methods are essential ingredients to obtain exact correlation functions uniquely for the LFT [5], the $N=1$ SLFT [6], and their boundary extensions [12, 13, 14, 15].

Differently from its simpler relatives, the $N=2$ SLFT is not renormalized and no duality appears. This nonrenormalization is a general aspect of $N=2$ supersymmetric quantum field theories in two-dimensional space-time. Without the duality, the functional relations satisfied by the correlation functions can not be solved uniquely. In [18, an $N=2$ super-CFT has been proposed as a dual theory to the $N=2$ SLFT under a transformation $b \rightarrow 1 / b$.. Based on this conjecture, the bulk two-point functions, or "reflection amplitudes", of both Neveu-Schwarz (NS) and Ramond (R) sectors have been computed and various independent checks have been made.

Computing the one-point functions of the $N=2 \mathrm{SLFT}$ is more complicated. The standard approach for one-point functions initiated by [12, 13] and followed by [12, 14, 15] is the conformal bootstrap method which can generate functional relations using the conformally invariant boundary actions as boundary screening operators. The boundary action of the $N=2$ SLFT has been recently derived in [19]. However, the 
lack of the duality prohibits this approach here too. Namely, the $N=2$ SLFT with this boundary action is not self-dual either and one needs to know the boundary action of the dual $N=2$ theory. Without this, one can not solve the functional relations uniquely. Due to the nonlocality of the bulk action of the dual $N=2$ theory [18, the method used in the $N=2$ SLFT [19] seems not be applicable. We need a different approach. In the previous works [13, 14, 15], one-point functions have been obtained from the conformal bootstrap methods and confirmed by modular transformation properties using a known relationship to the conformal boundary states [20]. In this paper we reverse the steps; we first derive the one-point functions from the modular transformation properties. Then, we relate them to the bulk and boundary actions of the $N=2$ SLFT and its dual theory by conformal bootstrap methods. Although the functional relations obtained in this way is not complete due to the limitation we already mentioned, they can give essential informations which the modular transformations can not provide.

There appeared already a paper which used the modular transformation method to find the one-point functions and associated boundary states [11. However, this paper considers only a special value of coupling constant and only a vacuum BC. Moreover, a direct relation to the $N=2$ SLFT is missing. Our results include general one-point functions for general BCs parametrized by a continuous parameter. We also provide various consistency checks. Using the one-point functions, we rederive the bulk reflection amplitudes and compare them with those derived independently [21, 18. Also we provide semiclassical checks. Furthermore, as previously described, we provide conformal boostrap analysis based on the $N=2$ SLFT and its dual theories and confirme the one-point functions obtained from the modular transformations are consistent with the bulk and boundary actions. As byproducts, we obtain a relation between the continuous BC parameter and the boundary cosmological constants of the two dual theories.

This paper is organized as follows. In sect.2 we introduce the bulk and boundary actions of the $N=2 \mathrm{SLFT}$ along with notations. Then, we derive the characters and their modular transformations in sect.3. In sect.4, we present our main results, the one-point functions and functional relations from the conformal bootstrap along with several consistency checks. We discuss a relation to boundary two-point functions and some concluding remarks in sect.5. 


\section{$2 \quad N=2$ Super-Liouville Theory}

The action of the $N=2$ SLFT with the boundary is given by

$$
\begin{aligned}
S= & \int d^{2} z\left[\frac{1}{2 \pi}\left(\partial \phi^{-} \bar{\partial} \phi^{+}+\partial \phi^{+} \bar{\partial} \phi^{-}+\psi^{-} \bar{\partial} \psi^{+}+\psi^{+} \bar{\partial} \psi^{-}+\bar{\psi}^{-} \partial \bar{\psi}^{+}+\bar{\psi}^{+} \partial \bar{\psi}^{-}\right)\right. \\
& \left.+i \mu b^{2} \psi^{-} \bar{\psi}^{-} e^{b \phi^{+}}+i \mu b^{2} \psi^{+} \bar{\psi}^{+} e^{b \phi^{-}}+\pi \mu^{2} b^{2} e^{b\left(\phi^{+}+\phi^{-}\right)}\right]+S_{B}
\end{aligned}
$$

where the boundary action is derived to be [19]

$$
\begin{aligned}
S_{B} & =\int_{-\infty}^{\infty} d x\left[-\frac{i}{4 \pi}\left(\bar{\psi}^{+} \psi^{-}+\bar{\psi}^{-} \psi^{+}\right)+\frac{1}{2} a^{-} \partial_{x} a^{+}\right. \\
& -\frac{1}{2} e^{b \phi^{+} / 2}\left(\mu_{B} a^{+}+\frac{\mu b^{2}}{4 \mu_{B}} a^{-}\right)\left(\psi^{-}+\bar{\psi}^{-}\right)-\frac{1}{2} e^{b \phi^{-} / 2}\left(\mu_{B} a^{-}+\frac{\mu b^{2}}{4 \mu_{B}} a^{+}\right)\left(\psi^{+}+\bar{\psi}^{+}\right) \\
& \left.-\frac{2}{b^{2}}\left(\mu_{B}^{2}+\frac{\mu^{2} b^{4}}{16 \mu_{B}^{2}}\right) e^{b\left(\phi^{+}+\phi^{-}\right) / 2}\right] .
\end{aligned}
$$

As in the LFT and the $N=1 \mathrm{SLFT}$, one should introduce a background charge $1 / b$ so that the interaction terms in Eq.(2.1) become the screening operators of the conformal field theory (CFT). As mentioned earlier, this background charge is unrenormalized due to the $N=2$ supersymmetry and the $N=2$ SLFT is not self-dual.

The stress tensor $T$, the supercurrent $G^{ \pm}$and the $U(1)$ current $J$ are given by

$$
\begin{aligned}
& T=-\partial \phi^{-} \partial \phi^{+}-\frac{1}{2}\left(\psi^{-} \partial \psi^{+}+\psi^{+} \partial \psi^{-}\right)+\frac{1}{2 b}\left(\partial^{2} \phi^{+}+\partial^{2} \phi^{-}\right), \\
& G^{ \pm}=\sqrt{2} i\left(\psi^{ \pm} \partial \phi^{ \pm}-\frac{1}{b} \partial \psi^{ \pm}\right), \quad J=-\psi^{-} \psi^{+}+\frac{1}{b}\left(\partial \phi^{+}-\partial \phi^{-}\right) .
\end{aligned}
$$

Using the mode expansions for the currents and their operator product expansion, one can find the $N=2$ super-Virasoro algebra

$$
\begin{aligned}
{\left[L_{m}, G_{r}^{ \pm}\right] } & =\left(\frac{m}{2}-r\right) G_{m+r}^{ \pm}, \quad\left[J_{n}, G_{r}^{ \pm}\right]= \pm G_{n+r}^{ \pm}, \\
\left\{G_{r}^{+}, G_{s}^{-}\right\} & =2 L_{r+s}+(r-s) J_{r+s}+\frac{c}{3}\left(r^{2}-\frac{1}{4}\right) \delta_{r+s}, \quad\left\{G_{r}^{ \pm}, G_{s}^{ \pm}\right\}=0, \\
{\left[L_{m}, J_{n}\right] } & =-n J_{m+n}, \quad\left[J_{m}, J_{n}\right]=\frac{c}{3} m \delta_{m+n},
\end{aligned}
$$

with the central charge

$$
c=3+6 / b^{2} .
$$

Due to anti-periodicity for the (NS) sector, the fermionic modes are given by halfintegers while for the $(\mathrm{R})$ sector they are integer modes due to the periodicity. 
The primary fields of the $N=2$ SLFT are classified into the (NS) and the (R) sectors and can be written in terms of the component fields as follows 22]:

$$
N_{\alpha \bar{\alpha}}=e^{\alpha \phi^{+}+\bar{\alpha} \phi^{-}}, \quad R_{\alpha \bar{\alpha}}^{ \pm}=\sigma^{ \pm} e^{\alpha \phi^{+}+\bar{\alpha} \phi^{-}},
$$

where $\sigma^{ \pm}$is the spin operators.

The conformal dimensions and the $U(1)$ charges of the primary fields $N_{\alpha \bar{\alpha}}$ and $R_{\alpha \bar{\alpha}}^{ \pm}$ can be obtained:

$$
\Delta_{\alpha \bar{\alpha}}^{N S}=-\alpha \bar{\alpha}+\frac{1}{2 b}(\alpha+\bar{\alpha}), \quad \Delta_{\alpha \bar{\alpha}}^{R}=\Delta_{\alpha \bar{\alpha}}^{N S}+\frac{1}{8},
$$

and

$$
\omega=\frac{1}{b}(\alpha-\bar{\alpha}), \quad \omega^{ \pm}=\omega \pm \frac{1}{2} .
$$

It is more convenient to use a 'momentum' defined by

$$
\alpha+\bar{\alpha}=\frac{1}{b}+2 i P,
$$

and the $U(1)$ charge $\omega$ instead of $\alpha, \bar{\alpha}$. In terms of these, the conformal dimensions are given by

$$
\Delta^{N S}=\frac{1}{4 b^{2}}+P^{2}+\frac{b^{2} \omega^{2}}{4} .
$$

From now on, we will denote a (NS) primary state by $|[P, \omega]\rangle$ and a $(\mathrm{R})$ state by $|[P, \omega, \epsilon]\rangle$ with $\epsilon= \pm 1$.

One can notice that the conformal dimenions and $U(1)$ charges are invariant under

$$
\alpha \rightarrow 1 / b-\bar{\alpha}, \quad \bar{\alpha} \rightarrow 1 / b-\alpha .
$$

This means $N_{\alpha \bar{\alpha}}$ should be identified with $N_{1 / b-\alpha, 1 / b-\bar{\alpha}}$ and similarly, for the (R) primary fields, up to normalization factors. In terms of the momentum parameter, this means an invariance under $P \rightarrow-P$. In semiclassical picture where the primary fields can be described by plane waves with momentum $P$ in the bosonic zero-mode space, this relation would imply that the wave with a momentum $P$ is reflected off from the potential wall and changes the momentum to $-P$. This qualitative description can be extended to the full quantum region where the exact reflection amplitudes are defined and computed using the functional relation methods. We will be back to this issue in sect.4.

\section{Characters and Modular Transformations}

A character is defined by the following trace over all the conformal states built on a specific primary state:

$$
\chi_{h}(q, y, t)=e^{2 \pi i k t} \operatorname{Tr}\left[q^{L_{0}-c / 24} y^{J_{0}}\right] .
$$


Here $k$ is a fixed constant for a given CFT and we set $k=1+2 / b^{2}$ for the $N=2$ SLFT. In terms of the modular parameters given by

$$
q=e^{2 \pi i \tau}, \quad y=e^{2 \pi i \nu}, \quad q^{\prime}=e^{-2 \pi i / \tau}, \quad y^{\prime}=e^{2 \pi i \nu / \tau}
$$

the modular group $S L(2, \mathbf{Z})$ is generated by the two elements $T, S$

$$
T:(\tau, \nu, t) \rightarrow(\tau+1, \nu, t), \quad S:(\tau, \nu, t) \rightarrow\left(-1 / \tau, \nu / \tau, t-\nu^{2} / 2 \tau\right)
$$

While the character transforms simply under $T$

$$
\chi_{h}(\tau, \nu, t)=e^{2 \pi i(h-c / 24)} \chi_{h}(\tau, \nu, t),
$$

the characters transform under $S$ nontrivially and are expressed by the modular $S$ matrix:

$$
\chi_{h}\left(-1 / \tau, \nu / \tau, t-\nu^{2} / 2 \tau\right)=\sum_{h^{\prime}} \mathbf{S}_{h h^{\prime}} \chi_{h^{\prime}}(\tau, \nu, t)
$$

\section{1 $N=2$ SLFT Characters}

To compute the $N=2$ SLFT characters, one should classify all the decendents by acting the super-Virasoro generators on a highest weight state, excluding not independent states [23. If we denote a (NS) primary field by the momentum $P$ and $U(1)$ charge $\omega$, the decendents are given by

$$
\cdots L_{-2}^{n_{2}} L_{-1}^{n_{1}} \cdots J_{-2}^{m_{2}} J_{-1}^{m_{1}} \cdots G_{-3 / 2}^{+} \epsilon_{3 / 2}^{+} G_{-1 / 2}^{+} \epsilon_{1 / 2}^{+} \cdots G_{-3 / 2}^{-} \epsilon_{3 / 2}^{-} G_{-1 / 2}^{-} \epsilon_{1 / 2}^{-}|[P, \omega]\rangle
$$

where the exponents $n_{i}, m_{i}$ are arbitrary nonnegative integers and $\epsilon_{r}^{ \pm}=0,1$ since $G_{r}^{ \pm 2}=0$.

For generic values of $P, \omega$, the $N=2$ SLFT has no null states and the characters can be obtained by simply summing the states. Using the definition given above, the character is computed to be

$$
\begin{aligned}
\chi_{[P, \omega]}^{N S}(q, y, t) & =e^{2 \pi i k t} q^{-1 / 8+P^{2}+b^{2} \omega^{2} / 4} y^{\omega} \prod_{n=1}^{\infty} \frac{\left(1+y q^{n-1 / 2}\right)\left(1+y^{-1} q^{n-1 / 2}\right)}{\left(1-q^{n}\right)^{2}} \\
& =e^{2 \pi i k t} q^{P^{2}+b^{2} \omega^{2} / 4} y^{\omega} \frac{\theta_{00}(q, y)}{\eta(q)^{3}},
\end{aligned}
$$

where we have introduced standard elliptic functions in the second line

$$
\begin{aligned}
\eta(q) & =q^{1 / 24} \prod_{n=1}^{\infty}\left(1-q^{n}\right) \\
\theta_{00}(q, y) & =\prod_{n=1}^{\infty}\left[\left(1-q^{n}\right)\left(1+y q^{n-1 / 2}\right)\left(1+y^{-1} q^{n-1 / 2}\right)\right] .
\end{aligned}
$$


The denominator of Eq.(3.7) originates from the modes $L_{-n}$ and $J_{-n}$ and the numerators from $G_{-r}^{ \pm}$.

For the conformal BCs of super-CFTs, one needs to consider characters and associated Ishibashi states of the $\widetilde{(\mathrm{NS})}$ sectors $[24$. The $\widetilde{(\mathrm{NS})}$ characters are defined by by

$$
\chi_{h}^{\widetilde{N S}}(q, y, t)=e^{2 \pi i k t} \operatorname{Tr}\left[(-1)^{F} q^{L_{0}-c / 24} y^{J_{0}}\right] .
$$

For a $N=2$ SLFT primary field with $[P, \omega],(-1)^{F}$ term contributes -1 for those decendents with odd number of $G_{-r}^{ \pm}$. This effect can be efficiently incorporated into the character formula by shifting $y \rightarrow-y$ in the product. Therefore, the $\widetilde{(\mathrm{NS})}$ character is given by

$$
\chi_{[P, \omega]}^{\widetilde{N S}}(q, y, t)=e^{2 \pi i k t} q^{P^{2}+b^{2} \omega^{2} / 4} y^{\omega} \frac{\theta_{00}(q,-y)}{\eta(q)^{3}} .
$$

The characters of the $(\mathrm{R})$ sector are rather different. Decendents of a $(\mathrm{R})$ primary field with $[P, \omega, \epsilon]$ whose conformal dimension and charge are given in Eqs.(2.10) and (2.8) are constructed by acting $L_{-n}$ 's, $J_{-n}$ 's and $G_{-r}^{ \pm}$'s. While $n$ is any positive integer, one should be careful for $r$. As noticed in [23], the (R) primary states satisfy

$$
G_{0}^{ \pm}|[P, \omega, \pm]\rangle=0 .
$$

Therefore, the (R) decendent module can include an extra state $G_{0}^{ \pm}|[P, \omega, \mp]\rangle$ and its decendents, respectively. Including these at each value of $\epsilon$, one can find the (R) character

$$
\begin{aligned}
\chi_{[P, \omega, \epsilon]}^{R}(q, y, t) & =e^{2 \pi i k t} q^{P^{2}+b^{2} \omega^{2} / 4} y^{\omega+\epsilon / 2}\left(1+y^{-\epsilon}\right) \prod_{n=1}^{\infty} \frac{\left(1+y q^{n}\right)\left(1+y^{-1} q^{n}\right)}{\left(1-q^{n}\right)^{2}} \\
& =e^{2 \pi i k t} q^{P^{2}+b^{2} \omega^{2} / 4} y^{\omega} \frac{\theta_{10}(q, y)}{\eta(q)^{3}}
\end{aligned}
$$

where we introduce another elliptic function

$$
\theta_{10}(q, y)=\left(y^{1 / 2}+y^{-1 / 2}\right) q^{1 / 8} \prod_{n=1}^{\infty}\left[\left(1-q^{n}\right)\left(1+y q^{n}\right)\left(1+y^{-1} q^{n}\right)\right] .
$$

\subsection{Modular Transformations}

Here we consider only $S$ transformation $(q, y, t) \rightarrow\left(q^{\prime}, y^{\prime}, t^{\prime}\right)$ defined in Eq. (3.3) with $t^{\prime}=t-\nu^{2} / 2 \tau$. For irrational CFTs such as the SLFTs with infinite number of primary fields, the modular $S$-matrix will be indexed by continuous parameters and the summation will be replaced by integrations.

First, the modular transformation properties of the elliptic functions are wellknown:

$$
\frac{\theta_{00}\left(q^{\prime}, y^{\prime}\right)}{\eta\left(q^{\prime}\right)^{3}}=e^{\pi i \nu^{2} / \tau} \frac{i}{\tau} \frac{\theta_{00}(q, y)}{\eta(q)^{3}}, \quad \frac{\theta_{10}\left(q^{\prime}, y^{\prime}\right)}{\eta\left(q^{\prime}\right)^{3}}=e^{\pi i \nu^{2} / \tau} \frac{i}{\tau} \frac{\theta_{00}(q,-y)}{\eta(q)^{3}} .
$$


Using these and Gaussian integrals, we have found the following modular transformations:

$$
\begin{aligned}
& \chi_{[P, \omega]}^{N S}\left(q^{\prime}, y^{\prime}, t^{\prime}\right)=b \int_{-\infty}^{\infty} d P^{\prime} \int_{-\infty}^{\infty} d \omega^{\prime} \cos \left(4 \pi P P^{\prime}\right) e^{-\pi i b^{2} \omega \omega^{\prime}} \chi_{\left[P^{\prime}, \omega^{\prime}\right]}^{N S}(q, y, t), \\
& \chi_{[P, \omega]}^{\widetilde{N S}}\left(q^{\prime}, y^{\prime}, t^{\prime}\right)=b \int_{-\infty}^{\infty} d P^{\prime} \int_{-\infty}^{\infty} d \omega^{\prime} \cos \left(4 \pi P P^{\prime}\right) e^{-\pi i b^{2} \omega \omega^{\prime}} \chi_{\left[P^{\prime}, \omega^{\prime}, \epsilon\right]}^{R}(q, y, t), \\
& \chi_{[P, \omega, \epsilon]}^{R}\left(q^{\prime}, y^{\prime}, t^{\prime}\right)=b \int_{-\infty}^{\infty} d P^{\prime} \int_{-\infty}^{\infty} d \omega^{\prime} \cos \left(4 \pi P P^{\prime}\right) e^{-\pi i b^{2} \omega \omega^{\prime}} \chi_{\left[P^{\prime}, \omega^{\prime}\right]}^{\widetilde{N S}}(q, y, t) .
\end{aligned}
$$

\subsection{Chiral Primary Fields}

An interesting class of $N=2$ SLFT primary fields is a chiral (and antichiral) primary field defined by

$$
G_{-1 / 2}^{+}|[P, \omega]\rangle=0
$$

Antichiral fields are defined by $G_{-1 / 2}^{-}$. Since they are almost the same, we consider only the chiral fields. If a primary field is chiral, then it should satisfy

$$
G_{1 / 2}^{-} G_{-1 / 2}^{+}|[P, \omega]\rangle=\left(2 L_{0}-J_{0}\right)|[P, \omega]\rangle=0 \quad \rightarrow \quad 2 \Delta=\omega
$$

This means

$$
\frac{1}{4 b^{2}}+P^{2}+\frac{b^{2} \omega^{2}}{4}=\frac{\omega}{2} \quad \rightarrow \quad P=i\left(\frac{b \omega}{2}-\frac{1}{2 b}\right) .
$$

We denote $|\omega\rangle=|[P, \omega]\rangle$. All the decendent states of a chiral primary field including $G_{-1 / 2}^{+}$mode must be truncated from the Hilbert space. This means $\epsilon_{1 / 2}^{+}=0$ in Eq.(3.6).

The character of a (NS) chiral primary field, then, can be written as Eq.(3.7) except one difference that the term $\left(1+y q^{n-1 / 2}\right)$ starts from $n=2$ because the mode $G_{-1 / 2}^{+}$ does not contribute. This changes the character into

$$
\chi_{|\omega\rangle}^{N S}(q, y, t)=e^{2 \pi i k t} \frac{q^{-1 / 4 b^{2}}\left(y q^{1 / 2}\right)^{\omega}}{1+y q^{1 / 2}} \frac{\theta_{00}(q, y)}{\eta(q)^{3}} .
$$

Using the method introduced in [25], one can derive the modular transformation of the character as follows:

$$
\begin{aligned}
\chi_{|\omega\rangle}^{N S}\left(q^{\prime}, y^{\prime}, t^{\prime}\right) & =\frac{b}{2} \int_{-\infty}^{\infty} d P^{\prime} \int_{-\infty}^{\infty} d \omega^{\prime}\left[\frac{e^{-\pi i b^{2} \omega \omega^{\prime}} \cosh \left[2 \pi b P^{\prime}\left(\omega-1-\frac{1}{b^{2}}\right)\right]}{2 \cosh \left(\pi b P^{\prime}+\frac{\pi i b^{2} \omega^{\prime}}{2}\right) \cosh \left(\pi b P^{\prime}-\frac{\pi i b^{2} \omega^{\prime}}{2}\right)}\right. \\
& \left.+\frac{e^{-\pi i b^{2}(\omega-1) \omega^{\prime}} \cosh \left[2 \pi b P^{\prime}\left(\omega-\frac{1}{b^{2}}\right)\right]}{2 \cosh \left(\pi b P^{\prime}+\frac{\pi i b^{2} \omega^{\prime}}{2}\right) \cosh \left(\pi b P^{\prime}-\frac{\pi i b^{2} \omega^{\prime}}{2}\right)}\right] \chi_{\left[P^{\prime}, \omega^{\prime}\right]}^{N S}(q, y, t) \\
& +i \sum_{n \in \mathbb{Z}} \int_{\frac{1}{b^{2}}}^{1+\frac{1}{b^{2}}} d \omega^{\prime} e^{-\pi i\left(2 n \omega+\omega+\omega^{\prime}-\frac{1}{b^{2}}\right)} q^{\frac{k}{2} n^{2}} y^{k n} \chi_{\left|\omega^{\prime}\right\rangle}^{N S}\left(q, y q^{n}, t\right) .
\end{aligned}
$$

Similar formulae for the antichiral (NS) primary fields and (anti-) chiral fields of the (R) and other sectors can be obtained. 


\subsection{Identity (Vacuum) Operator}

As we will see shortly, the identity operator plays a very important role in our derivation of the one-point functions. Therefore, we need to derive the character of this. The vacuum state $|0\rangle=|[-i / 2 b, 0]\rangle$ satisfies

$$
G_{-1 / 2}^{ \pm}|0\rangle=0, \quad L_{-1}|0\rangle=0 .
$$

This means that allowed decendents are given by Eq.(3.6) with restrictions that $n_{1}=$ $\epsilon_{1 / 2}^{ \pm}=0$. After excluding these states, one can find the (NS) character is given by

$$
\chi_{|0\rangle}^{N S}(q, y, t)=e^{2 \pi i k t} \frac{q^{-1 / 4 b^{2}}(1-q)}{\left(1+y q^{1 / 2}\right)\left(1+y^{-1} q^{1 / 2}\right)} \frac{\theta_{00}(q, y)}{\eta(q)^{3}} .
$$

It is obvious that the two factors in the denominators arise in the same way as the chiral fields and the factor $1-q$ in numerator comes from deducting the null state at level 1. If expanding the "specialized" character in a power series of $q$, we obtain

$$
\chi_{|0\rangle}^{N S}(q, 1,0)=1+q+2 q^{3 / 2}+3 q^{2}+\ldots
$$

We can identify first few levels with explicit decendent states. As expected, the level $1 / 2$ states are all truncated out and there is only one state left at the level 1 , namely, $J_{-1}|0\rangle$. Two states at the level $3 / 2$ should be $G_{-3 / 2}^{ \pm}|0\rangle$ and three states at the level 2 are created by $L_{-2}, J_{-2}, J_{-1}^{2}$.

The modular transformation of Eq.(3.25) can be derived as before following [25]

$$
\begin{aligned}
\chi_{|0\rangle}^{N S}\left(q^{\prime}, y^{\prime}, t^{\prime}\right) & =\int_{-\infty}^{\infty} d P \int_{-\infty}^{\infty} d \omega \mathbf{S}_{N S}(P, \omega) \chi_{[P, \omega]}^{N S}(q, y, t) \\
& +2 \sum_{n \in \mathbb{Z}} \int_{\frac{1}{b^{2}}}^{1+\frac{1}{b^{2}}} d \omega^{\prime} \sin \left[\pi\left(\omega^{\prime}-\frac{1}{b^{2}}\right)\right] q^{\frac{k}{2} n^{2}} y^{k n} \chi_{\left|\omega^{\prime}\right\rangle}^{N S}\left(q, y q^{n}, t\right), \\
\mathbf{S}_{N S}(P, \omega) & =\frac{\sinh (2 \pi b P) \sinh \left(\frac{2 \pi P}{b}\right)}{2 b^{-1} \cosh \left(\pi b P+\frac{i \pi b^{2} \omega}{2}\right) \cosh \left(\pi b P-\frac{i \pi b^{2} \omega}{2}\right)} .
\end{aligned}
$$

The first terms in Eqs. (3.233.27), which are of our main concern, can be also derived by expanding the denominators of Eqs.(3.22 3.25), applying Eq.(3.16), and resumming formally the infinite terms. However, this geometric sum can diverge and misses the contributions from the chiral primary characters. ${ }^{4}$ As explained in [26], these parts are necessary and have physical meanings with respect to the spectral flows of the superconformal field theories [27].

We will need to know the modular transformation of the identity operator in the $(\widetilde{N S})$ sector in the next section. For this, the Hilbert space of the conformal tower of

\footnotetext{
${ }^{4}$ We thank the authors of [26] for pointing this out.
} 
the identity operator is the same as the (NS) sector. As explained previously, the basic difference in this character arises from the $(-1)^{F}$ which changes $y \rightarrow-y$ in effect. Therefore, the character is given by

$$
\chi_{|0\rangle}^{\widetilde{N S}}(q, y, t)=e^{2 \pi i k t} \frac{q^{-1 / 4 b^{2}}(1-q)}{\left(1-y q^{1 / 2}\right)\left(1-y^{-1} q^{1 / 2}\right)} \frac{\theta_{00}(q,-y)}{\eta(q)^{3}} .
$$

Using the previous method [25], one can find the modular transformation

$$
\begin{aligned}
\chi_{|0\rangle}^{\widetilde{N S}}\left(q^{\prime}, y^{\prime}, t^{\prime}\right) & =\int_{-\infty}^{\infty} d P \int_{-\infty}^{\infty} d \omega \mathbf{S}_{R}(P, \omega) \chi_{[P, \omega, \epsilon]}^{R}(q, y, t) \\
& +2 \sum_{n \in \mathbb{Z}+\frac{1}{2}} \int_{\frac{1}{b^{2}}}^{1+\frac{1}{b^{2}}} d \omega^{\prime} \sin \left[\pi\left(\omega^{\prime}-\frac{1}{b^{2}}\right)\right] q^{\frac{k}{2}\left(n^{2}-\frac{1}{4}\right)} y^{k\left(n-\frac{1}{2}\right)} \chi_{\left|\omega^{\prime}\right\rangle}^{N S}\left(q, y q^{n}, t\right) \\
\mathbf{S}_{R}(P, \omega) & =\frac{\sinh (2 \pi b P) \sinh \left(\frac{2 \pi P}{b}\right)}{2 b^{-1} \sinh \left(\pi b P+\frac{i \pi b^{2} \omega}{2}\right) \sinh \left(\pi b P-\frac{i \pi b^{2} \omega}{2}\right)}
\end{aligned}
$$

\section{One-Point Functions in the Presence of Bound- ary}

In this section, we compute exact one-point functions of the (NS) and (R) bulk operators $N_{\alpha \bar{\alpha}}$ and $R_{\alpha \bar{\alpha}}^{\epsilon}$ of the $N=2$ SLFT with boundary. The one-point functions are defined by

$$
\left\langle N_{\alpha \bar{\alpha}}(\xi, \bar{\xi})\right\rangle=\frac{U^{N S}(\alpha, \bar{\alpha})}{|\xi-\bar{\xi}|^{2 \Delta_{\alpha \bar{\alpha}}^{N S}}}, \quad \text { and } \quad\left\langle R_{\alpha \bar{\alpha}}^{\epsilon}(\xi, \bar{\xi})\right\rangle=\frac{U^{R}(\alpha, \bar{\alpha})}{|\xi-\bar{\xi}|^{2 \Delta_{\alpha \bar{\alpha}}^{R}}}
$$

with the conformal dimensions given in Eq.(2.7). We will simply refer to the coefficients $U^{N S}(\alpha, \bar{\alpha})$ and $U^{R}(\alpha, \bar{\alpha})$ as the one-point functions.

\subsection{Vacuum Boundary Condition}

According to Cardy's formalism, one can associate a conformal BC with each primary state [8]. For the $N=2 \mathrm{SLFT}$, there will be infinite number of conformal BCs. These BCs can be constructed by the fusion process and related to the one-point functions. Let us begin with the 'vacuum' BC which corresponds to the identity operator. First we introduce an amplitude as an inner product between the Isibashi state of a primary state and the conformal boundary state ${ }^{5}$

$$
\left.\Psi_{\mathbf{0}}^{N S}(P, \omega)=\langle(\mathbf{0}) \mid[P, \omega]\rangle\right\rangle .
$$

\footnotetext{
${ }^{5}$ We denote a conformal BC in 'bold face' like $\mathbf{0}$ and a conformal boundary state like $|(\mathbf{0})\rangle$.
} 
From the modular transformation Eq.(3.27), the amplitude satisfies the following relation:

$$
\Psi_{\mathbf{0}}^{N S}(P, \omega) \Psi_{\mathbf{0}}^{N S^{\dagger}}(P, \omega)=\mathbf{S}_{N S}(P, \omega) .
$$

Since $\Psi_{\mathbf{0}}^{N S^{\dagger}}(P, \omega)=\Psi_{\mathbf{0}}^{N S}(-P, \omega)$, one can solve this up to some unknown constant as follows:

$$
\Psi_{\mathbf{0}}^{N S}(P, \omega)=\sqrt{\frac{b^{3}}{2}}\left(X_{N S}\right)^{\frac{i P}{b}} \frac{\Gamma\left(\frac{1}{2}-i b P+\frac{b^{2} \omega}{2}\right) \Gamma\left(\frac{1}{2}-i b P-\frac{b^{2} \omega}{2}\right)}{\Gamma\left(-\frac{2 i P}{b}\right) \Gamma(1-2 i b P)} .
$$

The unknown constant $X_{N S}$ does not depend on $P, \omega$ and can not be determined by the modular transformation alone. We will derive this constant later in this section by comparing with the bulk reflection amplitudes.

Similarly, for the (R) sector, we define the (R) amplitude by

$$
\left.\Psi_{\mathbf{0}}^{R}(P, \omega)=\langle(\mathbf{0}) \mid[P, \omega, \epsilon]\rangle\right\rangle
$$

which satisfies from Eq.(3.30)

$$
\Psi_{\mathbf{0}}^{R}(P, \omega) \Psi_{\mathbf{0}}^{R^{\dagger}}(P, \omega)=\mathbf{S}_{R}(P, \omega)
$$

The solution is up to a unknown constant:

$$
\Psi_{\mathbf{0}}^{R}(P, \omega)=-i \sqrt{\frac{b^{3}}{2}}\left(X_{R}\right)^{\frac{i P}{b}} \frac{\Gamma\left(-i b P+\frac{b^{2} \omega}{2}\right) \Gamma\left(1-i b P-\frac{b^{2} \omega}{2}\right)}{\Gamma\left(-\frac{2 i P}{b}\right) \Gamma(1-2 i b P)} .
$$

Again, the unknown constant $X_{R}$ will be fixed later.

\subsection{Continuous Boundary Condition}

Now we consider a continuous BC associated with a primary field. This field should be (NS) and its $U(1)$ charge should be zero because only the boundary neutral operators should appear. So, we consider the character of a (NS) primary state $|s\rangle \equiv|[s, 0]\rangle$ and its modular transformation. The parameter $s$ depends on the boundary parameter $\mu_{B}$ in Eq.(2.2). In this case Eq.(3.16) becomes

$$
\chi_{|s\rangle}^{N S}\left(q^{\prime}, y^{\prime}, t^{\prime}\right)=b \int_{-\infty}^{\infty} d P \int_{-\infty}^{\infty} d \omega \cos (4 \pi s P) \chi_{[P, \omega]}^{N S}(q, y, t) .
$$

Now following previous analysis of the modular transformation, this character should be written as

$$
\chi_{|s\rangle}^{N S}\left(q^{\prime}, y^{\prime}, t^{\prime}\right)=\int_{-\infty}^{\infty} d P \int_{-\infty}^{\infty} d \omega \Psi_{\mathbf{s}}^{N S}(P, \omega) \Psi_{\mathbf{0}}^{N S^{\dagger}}(P, \omega) \chi_{[P, \omega]}^{N S}(q, y, t) .
$$


Here we have defined an inner product between the conformal boundary state and an Ishibashi state

$$
\left.\Psi_{\mathbf{s}}^{N S}(P, \omega)=\langle(\mathbf{s}) \mid[P, \omega]\rangle\right\rangle .
$$

From Eqs.(4.8) and (4.9), one can find

$$
\Psi_{\mathbf{s}}^{N S}(P, \omega) \Psi_{\mathbf{0}}^{N S^{\dagger}}(P, \omega)=b \cos (4 \pi s P) .
$$

Now acting $\Psi_{\mathbf{0}}^{N S}(P, \omega)$ on this and using Eq.(4.3), we obtain

$$
\begin{aligned}
\Psi_{\mathbf{s}}^{N S}(P, \omega) & =b \Psi_{\mathbf{0}}^{N S}(P, \omega) \frac{\cos (4 \pi s P)}{\mathbf{S}_{N S}(P, \omega)} \\
& =\sqrt{2 b^{3}}\left(X_{N S}\right)^{\frac{i P}{b}} \frac{\Gamma\left(1+\frac{2 i P}{b}\right) \Gamma(2 i b P) \cos (4 \pi s P)}{\Gamma\left(\frac{1}{2}+i b P+\frac{b^{2} \omega}{2}\right) \Gamma\left(\frac{1}{2}+i b P-\frac{b^{2} \omega}{2}\right)} .
\end{aligned}
$$

One can follow the same step for the (R) sector. From Eq.(3.17) with $P=s, \omega=0$, one can find

$$
\Psi_{\mathbf{s}}^{R}(P, \omega) \Psi_{\mathbf{0}}^{R^{\dagger}}(P, \omega)=b \cos (4 \pi s P)
$$

where

$$
\left.\Psi_{\mathbf{s}}^{R}(P, \omega)=\langle(\mathbf{s}) \mid[P, \omega, \epsilon]\rangle\right\rangle .
$$

Using Eq.(4.6) on this, we can obtain

$$
\begin{aligned}
\Psi_{\mathbf{s}}^{R}(P, \omega) & =b \Psi_{0}^{R}(P, \omega) \frac{\cos (4 \pi s P)}{\mathbf{S}_{R}(P, \omega)} \\
& =-i \sqrt{2 b^{3}}\left(X_{R}\right)^{\frac{i P}{b}} \frac{\Gamma\left(1+\frac{2 i P}{b}\right) \Gamma(2 i b P) \cos (4 \pi s P)}{\Gamma\left(1+i b P-\frac{b^{2} \omega}{2}\right) \Gamma\left(i b P+\frac{b^{2} \omega}{2}\right)}
\end{aligned}
$$

The amplitudes (4.12) and (4.15) we have obtained are the one-point functions of the two sectors up to some normalization constants. To fix these constants, we recall the relation proved in 20]

$$
U_{\mathbf{k}}(\phi)=\frac{\langle(\mathbf{k}) \mid \phi\rangle\rangle}{\langle(\mathbf{k}) \mid 0\rangle\rangle}
$$

where $\mathbf{k}$ is a conformal $\mathrm{BC}, \phi$ a primary field, and $|\phi\rangle\rangle$, its Isibashi state. For the $N=2$ SLFT, this relation means

$$
U_{\mathbf{s}}^{N S}(P, \omega)=\frac{\Psi_{\mathbf{s}}^{N S}(P, \omega)}{\Psi_{\mathbf{s}}^{N S}(-i / 2 b, 0)}, \quad U_{\mathbf{s}}^{R}(P, \omega)=\frac{\Psi_{\mathbf{s}}^{R}(P, \omega)}{\Psi_{\mathbf{s}}^{N S}(-i / 2 b, 0)} .
$$

From Eqs.(4.12) and (4.15) we can obtain the one-point functions as follows:

$$
\begin{aligned}
U_{\mathbf{s}}^{N S}(P, \omega) & =\mathcal{N}\left(X_{N S}\right)^{\frac{i P}{b}} \frac{\Gamma\left(1+\frac{2 i P}{b}\right) \Gamma(2 i b P) \cos (4 \pi s P)}{\Gamma\left(\frac{1}{2}+i b P+\frac{b^{2} \omega}{2}\right) \Gamma\left(\frac{1}{2}+i b P-\frac{b^{2} \omega}{2}\right)}, \\
U_{\mathbf{s}}^{R}(P, \omega) & =\mathcal{N}\left(X_{R}\right)^{\frac{i P}{b}} \frac{\Gamma\left(1+\frac{2 i P}{b}\right) \Gamma(2 i b P) \cos (4 \pi s P)}{\Gamma\left(1+i b P-\frac{b^{2} \omega}{2}\right) \Gamma\left(i b P+\frac{b^{2} \omega}{2}\right)},
\end{aligned}
$$


where the normalization coefficient $\mathcal{N}$ can be fixed by

$$
U_{\mathbf{s}}^{N S}(-i / 2 b, 0)=1 \rightarrow \mathcal{N}=\left[\left(X_{N S}\right)^{1 / 2 b^{2}} \Gamma\left(1+b^{-2}\right) \cosh \left(\frac{2 \pi s}{b}\right)\right]^{-1}
$$

Then, from $U_{\mathbf{s}}^{R}(-i / 2 b, 0)=\left\langle\sigma^{ \pm}\right\rangle$, one can find

$$
\left\langle\sigma^{ \pm}\right\rangle=\frac{2}{\pi}\left(\frac{X_{R}}{X_{N S}}\right)^{1 / 2 b^{2}}
$$

The constants $X_{N S}$ and $X_{R}$ will be fixed shortly.

\subsection{Bulk Reflection Amplitudes}

The invariance of both conformal dimensions and $U(1)$ charges under Eq.(2.11) means that $N_{1 / b-\bar{\alpha}, 1 / b-\alpha}$ should be identified with $N_{\alpha \bar{\alpha}}$ and similarly for the (R) operators up to normalization factors. The reflection amplitudes are defined by two-point functions of the same operators

$$
\left\langle N_{\alpha \bar{\alpha}}(z, \bar{z}) N_{\alpha \bar{\alpha}}(0,0)\right\rangle=\frac{D^{N S}(\alpha, \bar{\alpha})}{|z|^{4 \Delta_{\alpha \bar{\alpha}}^{N S}}}, \quad\left\langle R_{\alpha \bar{\alpha}}^{+}(z, \bar{z}) R_{\alpha \bar{\alpha}}^{-}(0,0)\right\rangle=\frac{D^{R}(\alpha, \bar{\alpha})}{|z|^{4 \Delta_{\alpha \bar{\alpha}}^{R}}}
$$

with $\Delta_{\alpha \bar{\alpha}}^{N S}, \Delta_{\alpha \bar{\alpha}}^{R}$ given in Eq.(2.7). In general, identification of the two fields gives a relation

$$
\left\langle N_{\alpha \bar{\alpha}}(z, \bar{z}) \ldots\right\rangle=D^{N S}(\alpha, \bar{\alpha})\left\langle N_{\frac{1}{b}-\bar{\alpha}, \frac{1}{b}-\alpha}(z, \bar{z}) \ldots\right\rangle
$$

and similarly for the $(\mathrm{R})$ sector. Here the part ... can be any products of the primary fields.

It turns out that the computation of these quantities is much more complicated than that of the LFT or the $N=1$ SLFT case. As we mentioned earlier, the reason is the lack of the self-duality. In [18, the reflection amplitudes of the primary fields with zero $U(1)$ charges have been derived based on a conjectured $N=2$ super-CFT which is dual to the $N=2$ SLFT. While these results are based on the conjecture, the resulting reflection amplitudes have passed several consistency checks. Moreover, these results are in exact agreement with the reflection amplitudes which have been derived from certain integrable field theory with two parameters proposed in 28] which generates $N=2$ supersymmetry at special values of couplings [21]. This agreement between two independent approaches strongly supports the validity of the reflection amplitudes and the dual action.

Here, we provide another independent derivation of the reflection amplitudes based on the one-point functions we have derived. This computation will provide not only another confirmation of the results, but also can be used to fix the undetermined 
constants. The reflection relations among the correlation functions can be used for a simpliest case, namely, the one-point functions. In this case, the relation becomes

$$
\begin{aligned}
\left\langle N_{\alpha \bar{\alpha}}(z, \bar{z})\right\rangle & =D^{N S}(\alpha, \bar{\alpha})\left\langle N_{\frac{1}{b}-\bar{\alpha}, \frac{1}{b}-\alpha}(z, \bar{z})\right\rangle \\
\left\langle R_{\alpha \bar{\alpha}}(z, \bar{z})\right\rangle & =D^{R}(\alpha, \bar{\alpha})\left\langle R_{\frac{1}{b}-\bar{\alpha}, \frac{1}{b}-\alpha}(z, \bar{z})\right\rangle .
\end{aligned}
$$

These lead to the following equations:

$$
\frac{U_{\mathbf{s}}^{N S}(P, \omega)}{U_{\mathbf{s}}^{N S}(-P, \omega)}=D^{N S}(P, \omega), \quad \frac{U_{\mathbf{s}}^{R}(P, \omega)}{U_{\mathbf{s}}^{R}(-P, \omega)}=D^{R}(P, \omega) .
$$

For the neutral sector $\omega=0$, the reflection amplitudes has been derived in [18]

$$
\begin{aligned}
D^{N S}(P, 0) & =-\kappa^{-2 i P / b} \frac{\Gamma\left(1+\frac{2 i P}{b}\right)}{\Gamma\left(1-\frac{2 i P}{b}\right)} \frac{\Gamma(1+i P b)}{\Gamma(1-i P b)} \frac{\Gamma\left(\frac{1}{2}-i P b\right)}{\Gamma\left(\frac{1}{2}+i P b\right)}, \\
D^{R}(P, 0) & =\kappa^{-2 i P / b} \frac{\Gamma\left(1+\frac{2 i P}{b}\right)}{\Gamma\left(1-\frac{2 i P}{b}\right)} \frac{\Gamma(1-i P b)}{\Gamma(1+i P b)} \frac{\Gamma\left(\frac{1}{2}+i P b\right)}{\Gamma\left(\frac{1}{2}-i P b\right)} .
\end{aligned}
$$

where

$$
\kappa=\frac{\mu^{2} \pi^{2}}{2} \gamma\left(-b^{2}-1\right) \gamma\left(1+\frac{b^{2}}{2}\right) \gamma\left(\frac{b^{2}}{2}+\frac{3}{2}\right),
$$

with $\gamma(x)=\Gamma(x) / \Gamma(1-x)$ and the bulk cosmological constant $\mu$ in Eq.(2.1).

Inserting $\omega=0$ and using (4.18) and (4.19), the reflection amplitudes in Eq.(4.26) are indeed in exact agreement with Eqs.(4.27) and (4.28) if and only if we identify the constants

$$
X_{N S}=X_{R}=\left[2^{2 b^{2}} \kappa\right]^{-1}
$$

This provides a nontrivial check and completes our derivation for the one-point functions. Furthermore, we can use Eq.(4.26) to compute the reflection amplitudes for $\omega \neq 0$ case

$$
D^{N S}(P, \omega)=\left(2^{2 b^{2}} \kappa\right)^{-2 i P / b} \frac{\Gamma\left(1+\frac{2 i P}{b}\right)}{\Gamma\left(1-\frac{2 i P}{b}\right)} \frac{\Gamma(2 i b P)}{\Gamma(-2 i b P)} \frac{\Gamma\left(\frac{1}{2}-i b P+\frac{b^{2} \omega}{2}\right)}{\Gamma\left(\frac{1}{2}+i b P+\frac{b^{2} \omega}{2}\right)} \frac{\Gamma\left(\frac{1}{2}-i b P-\frac{b^{2} \omega}{2}\right)}{\Gamma\left(\frac{1}{2}+i b P-\frac{b^{2} \omega}{2}\right)}
$$

and

$$
D^{R}(P, \omega)=\left(2^{2 b^{2}} \kappa\right)^{-2 i P / b} \frac{\Gamma\left(1+\frac{2 i P}{b}\right)}{\Gamma\left(1-\frac{2 i P}{b}\right)} \frac{\Gamma(2 i b P)}{\Gamma(-2 i b P)} \frac{\Gamma\left(1-i b P-\frac{b^{2} \omega}{2}\right)}{\Gamma\left(1+i b P-\frac{b^{2} \omega}{2}\right)} \frac{\Gamma\left(-i b P+\frac{b^{2} \omega}{2}\right)}{\Gamma\left(i b P+\frac{b^{2} \omega}{2}\right)} .
$$

These results can be compared with those from the two-parameter family models [21] and we checked that two independent results match exactly.

To complete our derivation of the one-point functions, we should relate the boundary parameter $s$ with the boundary cosmological constant $\mu_{B}$ in Eq.(2.2). In principle, 
one should do this by deriving the functional relations following [12, 14, 15]. Solving these coupled equations, one can find the relation between the boundary parameters. However, without the boundary dual theory, this method does not work well for the $N=2$ SLFT. Instead, we analyze the pole structure of the one-point functions and compare them with direct calculations using the bulk and boundary action.

For this, we consider one-point function of a neutral (NS) field $N_{\alpha \alpha}$

$$
\text { residue }\left.\frac{U^{N S}(\alpha)}{\mathcal{N}}\right|_{\alpha=\left(b^{-1}-n b\right) / 2}=\left\langle e^{\alpha\left(\phi^{+}+\phi^{-}\right)}\right\rangle=\sum_{p, q} \frac{1}{p ! q !}\left\langle e^{\alpha\left(\phi^{+}+\phi^{-}\right)} V^{p} B^{q}\right\rangle_{0}
$$

where $V, B$ are the interaction terms in the bulk and boundary actions. If we choose $n=1(\alpha=1 / 2 b-b / 2)$, all terms vanish except $p=0, q=2$ which can be easily computed:

$$
\begin{aligned}
\left\langle e^{\alpha\left(\phi^{+}+\phi^{-}\right)}(i / 2) B^{2}\right\rangle_{0} & =-2 \bar{\mu}_{B}^{2} \int_{-\infty}^{\infty} d x_{1} d x_{2}\left|x_{1}-\frac{i}{2}\right|^{-2 \alpha b}\left|x_{2}-\frac{i}{2}\right|^{-2 \alpha b}\left|x_{1}-x_{2}\right|^{-\left(1+b^{2}\right)} \\
& =8 \pi \bar{\mu}_{B}{ }^{2} \Gamma\left(-b^{2}\right) \gamma\left(\frac{1+b^{2}}{2}\right) \sin \left(\pi \frac{1+b^{2}}{2}\right)
\end{aligned}
$$

with

$$
\bar{\mu}_{B}^{2}=\mu_{B}^{2}+\frac{\mu^{2} b^{4}}{16 \mu_{B}^{2}}
$$

The residue of Eq.(4.18) at $\alpha=\bar{\alpha}=1 / 2 b-b / 2$ becomes

$$
\frac{b}{2}\left(2^{2 b^{2}} \kappa\right)^{1 / 2} \frac{\Gamma\left(-b^{2}\right)}{\Gamma\left(\frac{1-b^{2}}{2}\right)^{2}} \cosh (2 \pi s b) .
$$

Comparing these two, we find

$$
\bar{\mu}_{B}^{2}=\frac{\mu b}{32 \pi} \cosh (2 \pi s b)
$$

\subsection{Conformal Bootstrap Approach}

The procedure to derive the functional equations satisfied by the one-point functions are identical to [12, 14, 15]. Consider two-point functions of neutral operators,

$$
G_{\alpha}^{N S}\left(\xi, \xi^{\prime}\right)=\left\langle R_{-\frac{1}{2 b}}^{+}(\xi) N_{\alpha}\left(\xi^{\prime}\right)\right\rangle, \quad G_{\alpha}^{R}\left(\xi, \xi^{\prime}\right)=\left\langle R_{-\frac{1}{2 b}}^{+}(\xi) R_{\alpha}^{-}\left(\xi^{\prime}\right)\right\rangle
$$

where $R_{-1 / 2 b}^{+}$is a degenerate $(\mathrm{R})$ operator, whose OPEs are given by

$$
\begin{aligned}
& R_{-\frac{1}{2 b}}^{+} N_{\alpha}=\left[R_{\alpha-\frac{1}{2 b}}^{+}\right]+C^{N S}(\alpha)\left[R_{\alpha+\frac{1}{2 b}}^{+}\right] \\
& R_{-\frac{1}{2 b}}^{+} R_{\alpha}^{-}=\left[N_{\alpha-\frac{1}{2 b}}\right]+C^{R}(\alpha)\left[N_{\alpha+\frac{1}{2 b}}\right] .
\end{aligned}
$$


Here the bracket [...] means the conformal tower of a given primary field and the structure constants have been computed in [18 based on the dual $N=2$ SLFT:

$$
\begin{aligned}
C^{N S}(\alpha) & =\tilde{\mu} \pi \gamma\left(1+b^{-2}\right) \frac{\Gamma\left(\frac{2 \alpha}{b}-\frac{1}{b^{2}}\right) \Gamma\left(1-\frac{2 \alpha}{b}\right)}{\Gamma\left(1-\frac{2 \alpha}{b}+\frac{1}{b^{2}}\right) \Gamma\left(\frac{2 \alpha}{b}\right)}, \\
C^{R}(\alpha) & =\tilde{\mu} \pi \gamma\left(1+b^{-2}\right) \frac{\Gamma\left(1+\frac{2 \alpha}{b}-\frac{1}{b^{2}}\right) \Gamma\left(-\frac{2 \alpha}{b}\right)}{\Gamma\left(-\frac{2 \alpha}{b}+\frac{1}{b^{2}}\right) \Gamma\left(1+\frac{2 \alpha}{b}\right)},
\end{aligned}
$$

where $\tilde{\mu}$, the cosmological constant of the dual theory, has been related to that of the $N=2$ SLFT in [18.

These two-point functions can be expressed as

$$
\begin{aligned}
G_{\alpha}^{N S}\left(\xi, \xi^{\prime}\right) & =U^{R}\left(\alpha-\frac{b}{2}\right) \mathcal{G}_{+}^{N S}\left(\xi, \xi^{\prime}\right)+C^{N S}(\alpha) U^{R}\left(\alpha+\frac{b}{2}\right) \mathcal{G}_{-}^{N S}\left(\xi, \xi^{\prime}\right) \\
G_{\alpha}^{R}\left(\xi, \xi^{\prime}\right) & =U^{N S}\left(\alpha-\frac{b}{2}\right) \mathcal{G}_{+}^{R}\left(\xi, \xi^{\prime}\right)+C^{R}(\alpha) U^{N S}\left(\alpha+\frac{b}{2}\right) \mathcal{G}_{-}^{R}\left(\xi, \xi^{\prime}\right)
\end{aligned}
$$

where $\mathcal{G}_{ \pm}\left(\xi, \xi^{\prime}\right)$ 's are expressed in terms of the special conformal blocks

$$
\mathcal{G}_{ \pm}^{N S}\left(\xi, \xi^{\prime}\right)=\frac{\left|\xi^{\prime}-\bar{\xi}^{\prime}\right|^{2 \Delta_{\alpha}^{N S}-2 \Delta_{-b / 2}^{R}}}{\left|\xi-\bar{\xi}^{\prime}\right|^{4 \Delta_{\alpha}^{N S}}} \mathcal{F}_{ \pm}^{N S}(\eta), \quad \mathcal{G}_{ \pm}^{R}\left(\xi, \xi^{\prime}\right)=\frac{\left|\xi^{\prime}-\bar{\xi}^{\prime}\right|^{2 \Delta_{\alpha}^{R}-2 \Delta_{-b / 2}^{N S}}}{\left|\xi-\bar{\xi}^{\prime}\right|^{4 \Delta_{\alpha}^{R}}} \mathcal{F}_{ \pm}^{R}(\eta)
$$

with

$$
\eta=\frac{\left(\xi-\xi^{\prime}\right)\left(\bar{\xi}-\bar{\xi}^{\prime}\right)}{\left(\xi-\bar{\xi}^{\prime}\right)\left(\bar{\xi}-\xi^{\prime}\right)}
$$

Here, the conformal blocks are given by the hypergeometric functions

$$
\begin{aligned}
\mathcal{F}_{+}^{N S}(\eta) & =\eta^{\frac{\alpha}{b}}(1-\eta)^{-\frac{1}{b^{2}}-\frac{1}{2}} F\left(\frac{2 \alpha}{b}, 1+\frac{1}{b^{2}} ; \frac{2 \alpha}{b}-\frac{1}{b^{2}}+1 ; \eta\right) \\
\mathcal{F}_{-}^{N S}(\eta) & =\eta^{\frac{\alpha}{b}}(1-\eta)^{-\frac{2}{b^{2}}+1} F\left(\frac{1}{b^{2}}, 1-\frac{2 \alpha}{b}+\frac{2}{b^{2}} ;-\frac{2 \alpha}{b}+\frac{1}{b^{2}}+1 ; \eta\right) \\
\mathcal{F}_{+}^{R}(\eta) & =\eta^{\frac{\alpha}{b}-\frac{1}{2}}(1-\eta)^{-\frac{1}{b^{2}}-\frac{1}{2}} F\left(\frac{2 \alpha}{b}+1, \frac{1}{b^{2}} ; \frac{2 \alpha}{b}-\frac{1}{b^{2}}+2 ; \eta\right) \\
\mathcal{F}_{-}^{R}(\eta) & =\eta^{\frac{\alpha}{b}-\frac{1}{2}}(1-\eta)^{-\frac{2}{b^{2}}+\frac{1}{2}} F\left(\frac{1}{b^{2}},-1-\frac{2 \alpha}{b}+\frac{2}{b^{2}} ;-\frac{2 \alpha}{b}+\frac{1}{b^{2}} ; \eta\right) .
\end{aligned}
$$

On the other hand, one can compute the two-point functions as both $R_{-1 / 2 b}^{+}$and $N_{\alpha}$ or $R_{\alpha}^{-}$approach on the boundary. The fusion of the degenerate operator with the boundary is described by a special bulk-boundary structure constant which could be computed as a boundary screening integral with one insertion of the boundary interaction of the dual $N=2$ theory if it were known. Since we can not fix it, we denote the unknown constant just as $\mathcal{R}(-1 / 2 b)$. Then, we can obtain the system of 
functional relations as follows:

$$
\begin{aligned}
\mathcal{R}\left(-\frac{1}{2 b}\right) U^{N S}(\alpha) & =\frac{\Gamma\left(1-\frac{1}{b^{2}}+\frac{2 \alpha}{b}\right) \Gamma\left(-\frac{2}{b^{2}}\right)}{\Gamma\left(1-\frac{2}{b^{2}}+\frac{2 \alpha}{b}\right) \Gamma\left(1-\frac{1}{b^{2}}\right)} U^{R}\left(\alpha-\frac{1}{2 b}\right) \\
& +C^{N S}(\alpha) \frac{\Gamma\left(1+\frac{1}{b^{2}}-\frac{2 \alpha}{b}\right) \Gamma\left(-\frac{2}{b^{2}}\right)}{\Gamma\left(1-\frac{2 \alpha}{b}\right) \Gamma\left(1-\frac{1}{b^{2}}\right)} U^{R}\left(\alpha+\frac{1}{2 b}\right) \\
\mathcal{R}\left(-\frac{1}{2 b}\right) U^{R}(\alpha) & =\frac{\Gamma\left(\frac{2 \alpha}{b}-\frac{1}{b^{2}}\right) \Gamma\left(-\frac{2}{b^{2}}\right)}{\Gamma\left(\frac{2 \alpha}{b}-\frac{2}{b^{2}}\right) \Gamma\left(1-\frac{1}{b^{2}}\right)} U^{N S}\left(\alpha-\frac{1}{2 b}\right) \\
& +C^{R}(\alpha) \frac{\Gamma\left(\frac{1}{b^{2}}-\frac{2 \alpha}{b}\right) \Gamma\left(-\frac{2}{b^{2}}\right)}{\Gamma\left(\frac{-2 \alpha}{b}\right) \Gamma\left(1-\frac{1}{b^{2}}\right)} U^{N S}\left(\alpha+\frac{1}{2 b}\right) .
\end{aligned}
$$

Although we do not know the bulk-boundary structure constant, we can elliminate it by taking ratio of above equations and find one relation which is complitely fixed. It can be shown that the one-point functions Eqs.(4.18) and (4.19) indeed satisfy this relation. This means not only that the one-point functions obtained from the modular bootstrap procedures are consistent with the $N=2$ SLFT actions, but also that the $N=2$ theory proposed in 18 is indeed dual to the $N=2$ SLFT. Furthermore, we can find the bulk-boundary structure constant as follows:

$$
\frac{\mathcal{R}\left(-\frac{1}{2 b}\right) \Gamma\left(1-\frac{1}{b^{2}}\right)}{\Gamma\left(-\frac{2}{b^{2}}\right) \sqrt{\tilde{\mu} \pi \gamma\left(1+\frac{1}{b^{2}}\right)}}=\cosh \left(\frac{2 \pi s}{b}\right) .
$$

Along with Eq.(4.37), this equation relates the boundary cosmological constant of the $N=2$ SLFT with that of the dual $N=2$ theory.

\subsection{Semiclassical Checks}

These results can be checked, semiclassically. As $b \rightarrow 0$, the $N=2$ SLFT (2.1) can be described by the Schrödinger equation

$$
\left[-\frac{1}{2} \frac{\partial^{2}}{\partial \Phi_{0}^{2}}+\frac{\pi^{2} \mu^{2} b^{2}}{8} e^{2 b \Phi_{0}}\right] \Psi_{P}\left(\Phi_{0}\right)=2 P^{2} \Psi_{P}\left(\Phi_{0}\right)
$$

where $\Phi_{0}=\left(\phi_{0}^{+}+\phi_{0}^{-}\right) / 2$ in terms of the zero-modes of $\phi^{ \pm}$. Solving this, one can derive the reflection amplitude

$$
D^{N S}(P, \omega)=-\left(\frac{\pi^{2} \mu^{2}}{16}\right)^{-2 i P / b} \frac{\Gamma\left(1+\frac{2 i P}{b}\right)}{\Gamma\left(1-\frac{2 i P}{b}\right)},
$$

and can show that this is consistent with Eq.(4.31).

Another interesting check is to compute the inner product semiclassically

$$
\langle(\mathbf{s}) \mid[P, \omega]\rangle=\int_{-\infty}^{\infty} d \Phi_{0} \Psi_{B_{s}}\left(\Phi_{0}\right) \Psi_{P}\left(\Phi_{0}\right)
$$


where the boundary state can be expressed by the boundary Lagrangian following [12]

$$
\Psi_{B_{s}}\left(\Phi_{0}\right)=\exp \left(-8 \pi^{2} b^{-1} \bar{\mu}_{B}^{2} e^{b \Phi_{0}}\right)
$$

with $\bar{\mu}_{B}$ a boundary cosmological constant defined in Eq.4.35). From this, one can find

$$
\langle(\mathbf{s}) \mid[P, \omega]\rangle=\left(\frac{8 \pi^{2} \bar{\mu}_{B}^{2}}{b}\right)^{-2 i P / b} \Gamma\left(\frac{2 i P}{b}\right),
$$

which, along with Eq.(4.37), agrees with Eq.(4.12).

\section{Discussions}

Using the one-point function, one can find a density of states which can be related to the boundary two-point functions. The partition function $Z_{s, s^{\prime}}^{N S}(q, y, t)$ with contiuous BCs on both boundaries parametrized by $s$ and $s^{\prime}$ can be obtained as

$$
Z_{s, s^{\prime}}^{N S}(q, y, t)=\int_{-\infty}^{\infty} d P \int_{-\infty}^{\infty} d \omega \chi_{[P, \omega]}^{N S}\left(q^{\prime}, y^{\prime}, t^{\prime}\right) \Psi_{\mathbf{s}}^{N S}(P) \Psi_{\mathbf{s}^{\prime}}^{N S^{\dagger}}(P, \omega)
$$

with the amplitude (4.12). This can be rewritten as

$$
\begin{aligned}
Z_{s, s^{\prime}}^{N S}(\tau) & =b \int_{-\infty}^{\infty} d P^{\prime} d \omega^{\prime} d P d \omega e^{-4 i \pi P P^{\prime}} e^{-i \pi b^{2} \omega \omega^{\prime}} \chi_{[P, \omega]}^{N S}(q, y, t) \Psi_{\mathbf{s}}^{N S}\left(P^{\prime}, \omega^{\prime}\right) \Psi_{\mathbf{s}^{\prime}}^{N S^{\dagger}}\left(P^{\prime}, \omega^{\prime}\right) \\
& =\int_{0}^{\infty} d P \int_{0}^{\infty} d \omega \chi_{[P, \omega]}^{N S}(q, y, t) \rho_{s s^{\prime}}^{N S}(P, \omega),
\end{aligned}
$$

where $\rho_{s s^{\prime}}^{N S}(P, \omega)$ is the density of states,

$$
\rho_{s s^{\prime}}^{N S}(P, \omega)=\frac{4}{\pi^{2}} \int_{-\infty}^{\infty} d u \int_{-\infty}^{\infty} d v\left[\frac{\cosh (b v)+\cos (u)}{2 \sinh (b v) \sinh (v / b)}\right] \cos (2 s v) \cos \left(2 s^{\prime} v\right) e^{-2 i v P-i u \omega}
$$

This quantity is not well-defined at $P=0$ and is to be properly regularized. This density of states is, on the other hand, conjectured to be related with the two-point function $d_{B}^{N S}\left(P, \omega \mid s, s^{\prime}\right)$ of boundary operator $n_{\alpha \bar{\alpha}}^{s s^{\prime}}$ by

$$
\rho_{s, s^{\prime}}^{N S}(P, \omega)=-\frac{i}{2 \pi} \frac{d}{d P} \log d_{B}^{N S}\left(P, \omega \mid s, s^{\prime}\right) .
$$

In this paper, we have derived one-point functions of the $N=2$ SLFT and provided various consistency checks. Our consistency checks also confirms the validity of the dual $N=2$ super-CFT conjecture in [18] and the boundary action proposed in [19]. It would be interesting to provide the boundary action for the dual theory so that one can complete the boundary bootstrap procedure for the $N=2$ SLFT with the boundary. Our result can be applied to $2 \mathrm{~d}$ superstring theories and related topics 
generalizing the work on the $N=1$ SLFT [10]. These results also can be used to study the integrable quantum field theories with $N=2$ supersymmetry which can be constructed as perturbed $N=2$ SLFT. We hope to report a progress in this direction in future publications.

\section{Note Added}

After finishing this article, we found a paper [26] which results overlap with ours in large part. While this paper deals mainly with the vacuum BC and applications and implications to the string theory, we are more interested in the one-point functions for a continuous $\mathrm{BC}$ in terms of the boundary cosmological constant and their relations to the conformal bootstrap equations.

\section{Acknowledgments}

We thank Jaemo Park for helpful discussions. This work was supported in part by Korea Research Foundation 2002-070-C00025. MS is supported by the Brain Pool program from Korean Association Science and Technology.

\section{References}

[1] A. Polyakov, Phys. Lett. B103 (1981) 207.

[2] T. Curtright and C. Thorn, Phys. Rev. Lett. 48 (1982) 1309.

[3] G.-L. Gervais, Comm. Math. Phys. 130 (1990) 252.

[4] J. Teschner, Phys. Lett. B363 (1995) 65.

[5] A. B. Zamolodchikov and Al. B. Zamolodchikov, Nucl. Phys. B477 (1996) 577.

[6] R. C. Rashkov and M. Stanishkov, Phys. Lett. B380 (1996) 49.

[7] R. H. Poghossian, Nucl.Phys. B496 (1997) 451.

[8] J. Cardy, Nucl. Phys. B240 (1984) 514.

[9] P. Lee, H. Ooguri, and J. Park, Nucl. Phys. B632 (2002) 283; B. Ponsot, V. Schomerus, and J. Teschner, JHEP 02 (2002) 016. 
[10] M.R. Douglas, I.R. Klebanov, D. Kutasov, J. Maldacena, E. Martinec and N. Seiberg, "A New Hat For The $c=1$ Matrix Model", hep-th/0307195.

[11] J. McGreevy, S. Murthy, and H. Verlinde, "Two-dimensional Superstrings and the Supersymmetric Matrix Model", hep-th/0308105.

[12] V. Fateev, A. Zamolodchikov and Al. Zamolodchikov, "Boundary Liouville Field Theory I. Boundary State and Boundary Two-point Function", hep-th/0001012.

[13] A. Zamolodchikov and Al. Zamolodchikov, "Liouville Field Theory on a Pseudosphere", hep-th/0101152.

[14] C. Ahn, C. Rim and M. Stanishkov, Nucl. Phys. B636 (2002) 497.

[15] T. Fukuda and K. Hosomichi, Nucl. Phys. B635 (2002) 215.

[16] K. Hori and A. Kapustin, JHEP 0108 (2002) 045.

[17] L. O'Raifeartaigh and V. V. Sreedhar, Phys. Lett. B461 (1999) 66.

[18] C. Ahn, C. Kim, C. Rim and M. Stanishkov, "Duality in N=2 Super-Liouville Theory", hep-th/0210208.

[19] C. Ahn and M. Yamamoto, "Boundary Action of $N=2$ Super-Liouville Theory", hep-th/0310046, to appear in Phys. Rev. D.

[20] J. Cardy and D. Lewellen, Phys. Lett. B259 (1991) 274.

[21] P. Baseilhac and V. Fateev, Nucl. Phys. B532 (1998) 567.

[22] G. Mussardo, G. Sotkov, and M. Stanishkov, Int. J. Mod. Phys. A4 (1989) 1135.

[23] W. Boucher, D. Friedan, and A. Kent, Phys. Lett. B172 (1986) 316; V. K. Dobrev, Phys. Lett. B186 (1987) 43.

[24] R. Nepomechie, J. Phys. A34 (2001) 6509.

[25] K. Miki, Int. J. Mod. Phys. A5 (1990) 1293.

[26] T. Eguchi and Y. Sugawara, "Modular Bootstrap for Boundary $N=2$ Liouville Theory", hep-th/0311141.

[27] T. Eguchi and A. Taormina, Phys. Lett. B200 (1988) 315; Phys. Lett. B210 (1988) 125.

[28] V. Fateev, Nucl. Phys. B473 (1996) 509. 